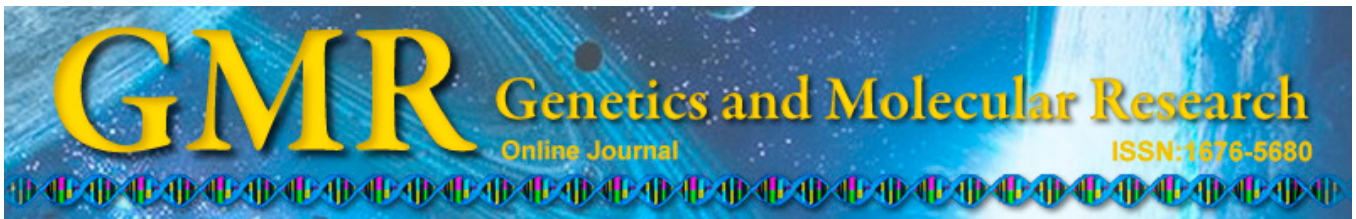

\title{
Positive selection sites in tertiary structure of Leguminosae Chalcone isomerase 1
}

\author{
R.K. Wang ${ }^{1}$, S.F. Zhan ${ }^{1}$, T.J. Zhao ${ }^{2}$, X.L. Zhou ${ }^{1}$ and C.E. Wang ${ }^{1}$ \\ ${ }^{1}$ Jiujiang Laboratory for Seed Quality of Soybean National Center for \\ Soybean Improvement, Research Center for Soybean, \\ College of Life Science, Jiujiang University, Jiangxi Province, China \\ ${ }^{2}$ National Key Laboratory for Crop Genetics and Germplasm Enhancement, \\ Soybean Research Institute, National Center for Soybean Improvement, \\ Nanjing Agricultural University, Jiangsu Province, China \\ Corresponding author: C.E. Wang \\ E-mail: soybeanw@163.com
}

Genet. Mol. Res. 14 (1): 1957-1967 (2015)

Received January 9, 2014

Accepted March 26, 2014

Published March 20, 2015

DOI http://dx.doi.org/10.4238/2015.March.20.5

ABSTRACT. Isoflavonoids and the related synthesis enzyme, chalcone isomerase 1 (CHI1), are unique in the Leguminosae, with diverse biological functions. Among the Leguminosae, the soybean is an important oil, protein crop, and model plant. In this study, we aimed to detect the generation pattern of Leguminosae CHII. Genome-wide sequence analysis of $\mathrm{CHI}$ in 3 Leguminosae and 3 other closely related model plants was performed; the expression levels of soybean chalcone isomerases were also analyzed. By comparing positively selected sites and their protein structures, we retrieved the evolution patterns for Leguminosae CHI1. A total of $28 \mathrm{CHI}$ and 7 FAP3 (CHI4) genes were identified and separated into 4 clades: CHI1, CHI2, CHI3, and FAP3. Soybean genes belonging to the same chalcone isomerase subfamily had similar expression patterns. CHI1, the unique chalcone isomerase subfamily in Leguminosae, showed signs of significant positive selection as well as special expression characteristics, indicating an accelerated evolution throughout its divergence. Eight sites were identified as 
undergoing positive selection with high confidence. When mapped onto the tertiary structure of $\mathrm{CHI} 1$, these 8 sites were observed surrounding the enzyme substrate only; some of them connected to the catalytic core of CHI. Thus, we inferred that the generation of Leguminosae CHI 1 is dependent on the positively selected amino acids surrounding its catalytic substrate. In other words, the evolution of CHI1 was driven by specific selection or processing conditions within the substrate.

Key words: Leguminosae; Soybean; Chalcone isomerase; Positive selection; Protein structure

\section{INTRODUCTION}

Isoflavonoids are secondary metabolites that mediate diverse biological functions and have significant ecological impacts. Plants use isoflavonoids and their derivatives as phytoalexin compounds targeting disease-causing pathogenic fungi and other microbes. In addition, the soybean uses isoflavonoids to stimulate soil-microbe rhizobium to form nitrogen-fixing root nodules (Verma, 1992). In the plant kingdom, approximately $95 \%$ of isoflavonoids are found in the family Leguminosae, and 60\% of Leguminosae flavonoids are 5-deoxyflavonoids (Hegnauer and Gpayer-Barkmeijer, 1993). The soybean is an important oil and protein crop, as well as a model plant of the Leguminosae. Soybean isoflavonoids have direct but complex effects on human health. In particular, they can prevent many hormone-dependent cancers and improve a woman's health (Ferrer et al., 2008).

Isoflavonoids are derived from the phenylpropanoid pathway, which is conserved in all plant species. In most species, chalcone synthase (CHS) and chalcone isomerase (CHI) are important in the production of isoflavonoids and flavonoids. Some CHIs isolated from Medicago and Arabidopsis have been studied structurally and mechanistically (Bednar and Hadcock 1988; Jez et al., 2000; Hur et al., 2004; Ngaki et al., 2012). These structure-function analyses suggest that the formation of a hydrogen bond network between the active site of $\mathrm{CHI}$ and its substrates is crucial for the catalytic activity of an enzyme (Jez and Noel, 2002; Hur et al., 2004). CHIs were derived from fatty-acid-binding proteins (FAP). Three CHI clades and one FAP3 clade, with distinctive phylogenic lineages, coexist in the soybean; they are $\mathrm{CHI}, \mathrm{CHI}, \mathrm{CHI} 3$, and FAP3 (previously termed CHI4 in some articles) (Ralston et al., 2005). CHI2 is ubiquitous in plants and converts 6'-deoxychalcone into (2S)-5-deoxychalcone. In contrast, CHI1 appears to be legume specific and possesses additional catalytic activity, which allows it to convert chalcone into (2S)-naringenin. (2S)-5-deoxychalcone and (2S)-naringenin are the precursors of many flavonoids and isoflavonoids. In vitro $\mathrm{CHI} 3$ has the similar produced as $\mathrm{CHI} 1$ and $\mathrm{CHI} 2$, but the function of CHI3 in plants has not been ascertained (Ralston, 2005). The FAP3 gene is highly homologous with $C H I$; however, their ligands in plants may be composed of different fatty acids. FAP3 knockout Arabidopsis thaliana plants show elevated $\alpha$-linolenic acid levels and marked reproductive defects, including aberrant seed formation (Ngaki et al., 2012).

Evolutionary biologists have conducted numerous studies on the factors affecting the evolutionary rates of protein-coding regions over the years (Ridout et al., 2010). Evolutionary rates vary not only between proteins but also between different sites within a single protein (Larracuente et al., 2008). The likelihood that positive selection alters an amino acid at a given site may depend on several factors such as the physical and chemical nature of the amino 
acid, the functional importance of the site, the surrounding environment, the physical properties of the structure, and the folding properties of the structure (Kosiol et al., 2008). Protein secondary structure, the physical arrangement of the amino acid chain, is produced mainly by the amino acid sequence and is another factor that may contribute to varying evolution rates at different amino acid positions. The amino acid order directly affects protein folding, and, therefore, tertiary structure and function; it is also highly conserved between homologous proteins (Petersen et al., 2007; Ridout et al., 2010).

The isoflavonoid and related CHII gene family are only found in the Leguminosae. In this paper, the positively selected sites of $C H I$ and $F A P 3$ sequences were compared among 3 Leguminosae and 3 other closely related plants to detect whether there are different evolution patterns among the Leguminosae CHII genes. This will provide a new insight into the specific genetic system of isoflavonoids in the Leguminosae and will be beneficial for soybean breeding.

\section{MATERIAL AND METHODS}

\section{Gene retrieval and identification}

Genome data for 6 model plants (i.e., Glycine max, Medicago truncatula, Phaseolus vulgaris, Arabidopsis thaliana, Ricinus communis, and Cucumis sativus) were used for sequence analyses. CHI proteins were searched on the Phytozome website (http://www.phytozome.net/) via BLASTP (E-value $<1 \mathrm{e}^{-50}$ ). The sequences collected were used for subsequent BLASTP searches to identify any missing sequences. Finally, all of the sequences identified were submitted to the Pfam database (http://pfam.sanger.ac.uk/search) (Punta et al., 2012) to check whether they contained a complete chalcone domain (the Pfam number of the chalcone isomerase is PF02431). Some genes were adjusted manually. All information for the CHI genes, including accession Nos., chromosomal locations, open reading frame lengths, and number of exons and introns, were retrieved from the Phytozome database. Structures of the genes and regions encoding the Pfam domains were created using the Gene Structure Display Server (http://gsds.cbi.pku.edu.cn/) (Guo et al., 2007).

\section{Phylogenetic and expansion pattern research}

The $\mathrm{CHI}$ candidate genes collected were used for phylogenetic analyses. Amino acid sequences were aligned using the CLUSTAL program of Molecular Evolutionary Genetics Analysis 4.0 (Tamura et al., 2007). The alignment results guided the neighbor-joining tree construction using the DNA sequences, and any alignment gaps were deleted manually. The parameters were set as follows: model, p-distance; bootstrap, 1000 replicates; and gap/missing data, pairwise deletion.

Expressed sequence tag (EST) resources have increased significantly for the soybean and may be used to research gene transcripts. The EST data can be used to detect the expansion pattern of a given gene family. In soybean, the identification of ESTs has proceeded rapidly, with approximately 1.5 million ESTs now available in GenBank (as of July 12, 2012). The EST data can be searched from GenBank (http://www.ncbi.nlm.nih.gov/dbEST/index. html) and well analyzed via web SoyKB (http://soykb.org/). 


\section{Molecular evolution and tertiary structure rebuilding}

We used a maximum likelihood approach to investigate positive selection, with a Codeml procedure via Phylogenetic Analysis by Maximum Likelihood 4.0 under branch-site model situations. Sites under positive selection will show a non-synonymous/synonymous rate ratio ( $\mathrm{dN} / \mathrm{dS}$, denoted as $\omega$ ) that differs from that of the other sites. The branch-site models allow $\omega$ to vary both between sites in the sequences and across branches on the tree. It assumes that there are 4 site classes in the sequence of a tree. The first class is highly conserved in all branches, with a small $\omega$ ratio (i.e., $\omega_{0}$ ). The second class includes neutral or weakly constrained sites with $\omega_{1}$, where $\omega_{1}$ is near 1 . In the third and fourth classes, the given branch is defined as foreground branches. The background lineages have $\omega_{0}$ or $\omega_{1}$, but the foreground branches have $\omega_{2}$, which may be $>1$. Its purpose is to detect positive selection that affects a few sites along given lineages (i.e., foreground branches). In the likelihood ratio test, the null hypothesis fixes $\omega_{2}=1$ (i.e., neutral selection), and the alternative hypothesis constrains $\omega_{2} \geq 1$ (i.e., positive selection). In the presence of positive selection, the posterior probabilities for the sites with positive selection were calculated using the Bayes empirical Bayes (BEB) method (Yang, 2007).

The predicted secondary structures for CHI were obtained using the UCL web site tools (http://bioinf.cs.ucl.ac.uk/psipred/). MtCHII is well studied (Jez et al., 2000; Ngaki et al., 2012), and it was acquired and analyzed from the protein data bank (http://www.rcsb.org/ $\mathrm{pdb} /$ home/home.do).

\section{RESULTS}

\section{Identification and organization of $\mathrm{CHI}$ genes}

Twenty-eight $C H I$ and 7 FAP3 genes were identified by BLAST from the 6 model plants. Notably, leguminous plants have more $C H I$ members than nonleguminous plants. There were always 6-8 genes encoding $\mathrm{CHI}$ in leguminous plants, while only 3-4 genes were found in the other plants. This is because the Leguminosae have additional copies of CHII (Table 1). Phylogenetic relationships between these genes were similar according to the neighbor-joining and Bayesian methods (data not shown). In agreement with previous studies, these genes could be divided into 4 clades: CHI1, CHI2, CHI3, and FAP3 (also known as CHI4 in some articles). The CHI3 and FAP3 genes were found in $M$. truncatula, possibly because of its low sequencing throughput.

The information from the phylogenetic tree and the exon/intron structure permitted exploration of the evolutionary relationships of the gene families. $C H I 1$ is a special isoflavonoid related subfamily only found in the Leguminosae, with 3, 2, or 5 members in G. max, $P$. vulgaris, and $M$. truncatula, respectively. The leguminous $C H I 1$ genes always contained 4 exons. MtCHIIB, MtCHIIC, and MtCHIID were divergent from the other CHIIs and contained 5,2 , or 3 exons, respectively. Moreover, they had no complete $C H I$ regions, possibly indicating a process of pseudogenization. A few copies were found as $C H I 2$ in these plants. CHII and $\mathrm{CHI} 2$ were identified as highly homologous genes, with similar exon/intron structures, untranslated regions, and $\mathrm{CHI}$ regions (Figure 1). Furthermore, genome sequence analysis revealed that $C H I 1$ and $C H I 2$ form a tandem cluster in the Leguminosae (Table 1). We also analyzed the sequence of FAP3, which is the ancestor gene subfamily of $C H I$ (Ngaki et al., 2012). The results of phylogenetic analyses were similar to those of Lyle Ralston (Ralston et al., 2005), which defined FAP3 as CHI4. 
Table 1. Candidate genes for $C H I$ and FAP3.

\begin{tabular}{|c|c|c|c|}
\hline Gene symbol & Local tag in phytozome & Gene length (bp) & E value in Pfam* \\
\hline AthCHI $2 A$ & AT3G55120 & 915 & $8.00 \mathrm{E}-55$ \\
\hline AthCHI $2 B$ & AT5G66220 & 2051 & $4.60 \mathrm{E}-39$ \\
\hline AthCHI 3 & AT1G53520 & 523 & $6.40 \mathrm{E}-54$ \\
\hline AthFAP 3 & AT5G05270 & 1540 & $8.70 \mathrm{E}-50$ \\
\hline $\mathrm{CsaCHI} 2$ & Cucsa. 182640 & 1356 & $7.40 \mathrm{E}-54$ \\
\hline CsaCHI 3 & Cucsa.058090 & 1687 & $4.60 \mathrm{E}-55$ \\
\hline CsaFAP 3 & Cucsa.143940 & 997 & $1.20 \mathrm{E}-47$ \\
\hline GmaCHI $1 \mathrm{~A}$ & Glyma20g38560 & 4930 & $1.30 \mathrm{E}-52$ \\
\hline GmaCHI $1 B 1$ & Glyma20g38570 & 3243 & $8.90 \mathrm{E}-58$ \\
\hline GmaCHI $1 B 2$ & Glyma10g43850 & 2900 & $1.20 \mathrm{E}-56$ \\
\hline GmaCHI 2 & Glyma20g38580 & 1964 & $4.90 \mathrm{E}-60$ \\
\hline GmaCHI $3 A$ & Glyma13g33730 & 1916 & $6.50 \mathrm{E}-53$ \\
\hline GmaCHI $3 B$ & Glyma15g39050 & 2630 & $1.70 \mathrm{E}-53$ \\
\hline GmaFAP $3 A$ & Glyma06g 14820 & 4118 & $5.20 \mathrm{E}-48$ \\
\hline GmaFAP $3 B$ & Glyma04g40030 & 4101 & $6.10 \mathrm{E}-47$ \\
\hline MtrCHI IAI & Medtrlg146040 & 2437 & $8.90 \mathrm{E}-51$ \\
\hline MtrCHI $1 A 2$ & Medtr1g146050 & 2071 & $2.50 \mathrm{E}-53$ \\
\hline MtrCHI $1 B$ & Medtr1g146060 & 1323 & $2.00 \mathrm{E}-06$ \\
\hline MtrCHI IC & Medtrlg146210 & 984 & $4.20 \mathrm{E}-02$ \\
\hline MtrCHI $1 D^{*}$ & Medtrlg146080 & 2932 & $1.70 \mathrm{E}-51$ \\
\hline MtrCHI $2 A$ & Medtrlg146030 & 1688 & $3.10 \mathrm{E}-59$ \\
\hline MtrCHI $2 B$ & Medtrlg146220 & 1846 & $5.50 \mathrm{E}-59$ \\
\hline PtrCHI 2 & POPTR_0010s21980 & 1390 & $2.30 \mathrm{E}-62$ \\
\hline PtrCHI $3 A$ & POPTR_0011s10330 & 777 & $5.30 \mathrm{E}-56$ \\
\hline PtrCHI $3 B$ & POPTR_0001s38970 & 1799 & $7.80 \mathrm{E}-40$ \\
\hline PtrFAP 3 & POPTR_0019s08610 & 1514 & $5.00 \mathrm{E}-46$ \\
\hline PvuCHI 1A & Phvulv091023708m.g & 2308 & $9.80 \mathrm{E}-53$ \\
\hline PvuCHI $1 \mathrm{~B}$ & Phvulv091005737m.g & 2296 & $1.40 \mathrm{E}-52$ \\
\hline PvuCHI $2 A$ & Phvulv091003077m.g & 1796 & $3.20 \mathrm{E}-61$ \\
\hline PvuCHI $2 B$ & Phvulv091005738m.g & 1867 & $5.80 \mathrm{E}-61$ \\
\hline PvuCHI 3 & Phvulv091004776m.g & 1744 & $2.20 \mathrm{E}-50$ \\
\hline PvUFAP 3 & Phvulv091026497m.g & 1721 & $1.60 \mathrm{E}-46$ \\
\hline RcoCHI 2 & $29740 . t 000017$ & 2722 & $1.90 \mathrm{E}-44$ \\
\hline RcoCHI 3 & 29989.t000018 & 4477 & $2.60 \mathrm{E}-29$ \\
\hline RCoFAP 3 & 29729.t000101 & 1857 & $6.10 \mathrm{E}-48$ \\
\hline
\end{tabular}

*The protein family identified is PF02431.10.

\section{Expression patterns of soybean chalcone isomerase}

EST databases contain libraries organized by organism, tissue type, and developmental stage that can provide valuable information for gene expression research. Thus, EST mining was used to analyze transcript levels of soybean chalcone isomerase in the leaf, flower, root, root nodule, pod, and seed. From the different tissue types and developmental stages, expression of all the soybean chalcone isomerase genes was detected. Specialized enzymes biosynthesize chemicals of given functions, often sharing a pedigree with primary metabolic enzymes (Hartmann, 2007). Genes belonging to a given isomerase subfamily always have similar expression patterns. The 3 copies of $\mathrm{GmCHII}$ were highly expressed in the root. Thus, we suggest that $G m C H I 1$ plays an important role in isoflavonoid synthesis in the root. Moreover, GmCHIIA was expressed at a higher level than that of GmCHIIB1 and GmCHIB2 in all tissues, suggesting its functional importance. Despite their close relationship, $\mathrm{GmCHIIB1}$ was typically expressed in the seed, while GmCHIIB2 was expressed in the pod shell. GmCHI2 and $\mathrm{GmCHI} 3$ were barely detected in the root or root nodule, and they shared similar tissue expression characteristics. This was different from the 2 GmFAP3 genes, which had high transcription in the seed and root. Thus, phylogenetically divergent genes also exhibited different expression patterns. 


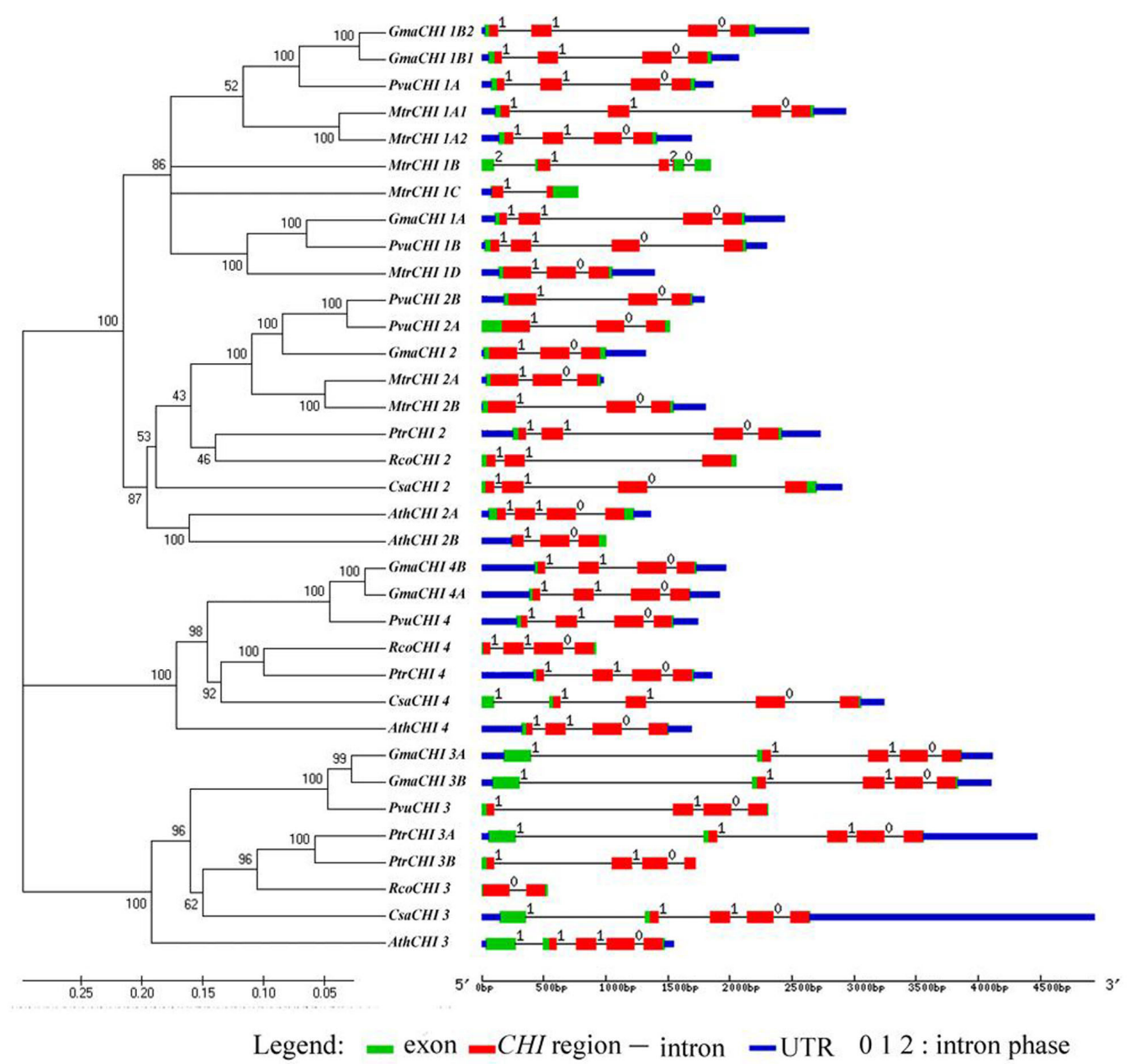

Figure 1. Neighbor-joining tree and gene structure of $C H I$ and FAP3. Sequences are shown by gene symbols. Bootstrap values from neighbor-joining analyses are listed to the left of each node. Values $>50$ are shown. Exons and introns are shown by filled boxes and single lines, respectively. The $C H I$ or $F A P 3$ region was identified from the Pfam database.

\section{Positively selected site detection in Leguminosae CHI}

To determine which sites differ in their evolutionary patterns, the $\omega$ values for sites were calculated using a branch-specific model. The 4 main clades of $C H I$ and FAP3 were independently defined as the foreground branches. When the CHI1, CHI2, and FAP3 branches were defined as the foreground branches, the null hypothesis was rejected $(\mathrm{P}<0.01)$, and the estimated parameters of the alternative hypotheses indicated that an average of $8-39 \%$ of the sites on these branches were under positive selection, with $\omega$ values of $\omega_{2}>1$ (Table 2). In the FAP3 branch $\left(\omega_{2}=999\right)$, some sites undergoing synonymous mutation did not contain nonsynonymous mutations, and the value of $\mathrm{dN} / \mathrm{dS}$ could not be calculated. A BEB analysis was used, rather than Naive Empirical Bayes, to identify putative codons under positive selection; 
Naive Empirical Bayes is less conservative and can be more prone to error in smaller data sets (Casola and Hahn., 2009).

In the branch-site model leading to CHII or FAP3, 28 or $39 \%$ of sites were under positive selection, respectively; and 8 or 10 sites were found in the BEB analysis, respectively, with a posterior probability of $>95 \%$ (Table 2). This suggests that many sites in CHII and FAP3 have undergone adaptive evolution. Considering that FAP3 is functionally different from $\mathrm{CHI}$, its abundant number of positively selected sites is not surprising. As a special protein existing only in Leguminosae, $C H I 1$ also has many positively selected sites indicating functional conversion. On the other hand, no sites were detected by BEB analysis in branch CHI2, and only $8 \%$ of sites were under positive selection in the estimated parameters of the branch-site model. Meanwhile, the alternative hypothesis of $\mathrm{CHI} 3$ could not reject the null hypothesis. This suggests that $\mathrm{CHI} 2$ and $\mathrm{CHI} 3$ have not undergone significant positive selection during their divergence from the other $\mathrm{CHI}$ genes.

Table 2. Summary statistics for the detection of selection using branch-site models.

\begin{tabular}{|c|c|c|c|c|}
\hline \multirow{2}{*}{$\begin{array}{l}\text { Foreground } \\
\text { branch }\end{array}$} & \multicolumn{2}{|c|}{ Null hypothesis } & \multicolumn{2}{|c|}{ Alternative hypothesis } \\
\hline & Estimated parameters & $\ln L$ & Estimated parameters & $\ln L$ \\
\hline $\mathrm{CHII}$ & $\begin{array}{c}\mathrm{P}_{0}=0.40, \mathrm{P}_{1}=0.05, \mathrm{P}_{2}+\mathrm{P}_{3}=0.56 \\
\omega_{0}=0.16, \omega_{1}=\omega_{2}=1\end{array}$ & -10667.13 & $\begin{array}{c}\mathrm{P}_{0}=0.64, \mathrm{P}_{1}=0.08, \mathrm{P}_{2}+\mathrm{P}_{3}=0.28 \\
\omega_{0}=0.16, \omega_{1}=1, \omega_{2}=14.69\end{array}$ & $-10663.67 * *$ \\
\hline $\mathrm{CHI} 2$ & $\begin{array}{c}\mathrm{P}_{0}=0.08, \mathrm{P}_{1}=0.0_{1}, \mathrm{P}_{2}+\mathrm{P}_{3}=0.91 \\
\omega_{0}=0.17, \omega_{1}=\omega_{2}=1\end{array}$ & -10674.08 & $\begin{array}{c}\mathrm{P}_{0}=0.81, \mathrm{P}_{1}=0.11, \mathrm{P}_{2}+\mathrm{P}_{3}=0.08 \\
\omega_{0}=0.16, \omega_{1}=1, \omega_{2}=999\end{array}$ & $-10667.46^{* *}$ \\
\hline $\mathrm{CHI3}$ & $\begin{array}{c}\mathrm{P}_{0}=0.58, \mathrm{P}_{1}=0.07, \mathrm{P}_{2}+\mathrm{P}_{3}=0.35 \\
\omega_{0}=0.17, \omega_{1}=\omega 2=1\end{array}$ & -10673.77 & $\begin{array}{c}P_{0}=0.56, P_{1}=0.07, P_{2}+P_{3}=0.37 \\
\omega_{0}=0.17, \omega_{1}=1, \omega_{2}=3.91\end{array}$ & -10672.79 \\
\hline FAP3 & $\begin{array}{c}\mathrm{P}_{0}=0.58, \mathrm{P}_{1}=0.07, \mathrm{P}_{2}+\mathrm{P}_{3}=0.35 \\
\omega_{0}=0.17, \omega_{1}=\omega_{2}=1\end{array}$ & -10671.51 & $\begin{array}{c}\mathrm{P}_{0}=0.54, \mathrm{P}_{1}=0.07, \mathrm{P}_{2}+\mathrm{P}_{3}=0.39 \\
\omega_{0}=0.17, \omega_{1}=1, \omega_{2}=999\end{array}$ & $-10663.33 * *$ \\
\hline
\end{tabular}

MtrCHI 1B, MtrCHI 1C, RcoCHI 2, AthCHI 2B, PtrCHI 3B, RcoCHI 3, were not included in the branch-specific model because its long gaps; 197 codons were used. The test statistic $2 \Delta l$ is compared to a $\chi^{2}$ distribution with 1 degree of freedom; **significant results.

\section{Secondary and tertiary structures of CHI1}

Understanding the structure of CHI and FAP3 is important for clarifying the roles of these proteins and providing insight into potential evolutionary pathways of CHIs. Highly similar $\alpha$-helix and $\beta$-sheet structures were observed for all of the CHI and FAP3 proteins examined, which always included $7 \alpha$-helixes and $7 \beta$-sheets in the same order (Figure 2a). Near these structural domains, conserved amino acid residues were found, implying their functional importance. Figure 2b shows the structure of MtCHI1 from the Protein Data Bank, which was derived from X-ray crystallography by Jez et al. (2000). The overall structure of MtCHI resembles an upside-down bouquet that adopts an open-faced $\beta$-sandwich fold; a large $\beta$-sheet ( $\beta 3 \mathrm{a}-\beta 3 \mathrm{f})$ and a layer of $\alpha$-helices $(\alpha 1-\alpha 7)$ comprise the core structure, with short $\beta$-strands $(\beta 1, \beta 2)$ on the opposite side of the large $\beta$-sheet (Jez et al., 2000; Ngaki et al., 2012).

We have detected the primary, positively selected sites in CHII and FAP3 (in the branch-site model, $\mathrm{CHI}$, $\mathrm{CHI}$, and $\mathrm{FAP} 3$ were under positive selection but no sites were identified via $\mathrm{BEB}$ analysis of $\mathrm{CHI2}$ ). Note that these sites were calculated based on the branches of all $C H I 1$ and FAP3 proteins rather than a single sequence. In the branch leading to $C H I 1,8$ selection sites were found ( $95 \%$ level of $\mathrm{BEB})$, which were always in the regions of $\beta 3 \mathrm{a}, \beta 3 \mathrm{c}$, $\beta 3 \mathrm{e}, \alpha 7$, and, especially, $\alpha 4$, indicating functionally accelerated evolution of these secondary 
structures. FAP3, $\beta 3 \mathrm{a}, \beta 3 \mathrm{~d}, \beta 3 \mathrm{f}$, and $\alpha 6$ were found to contain positively selected sites. Domains containing positively selected sites usually have a significant impact on protein evolution.

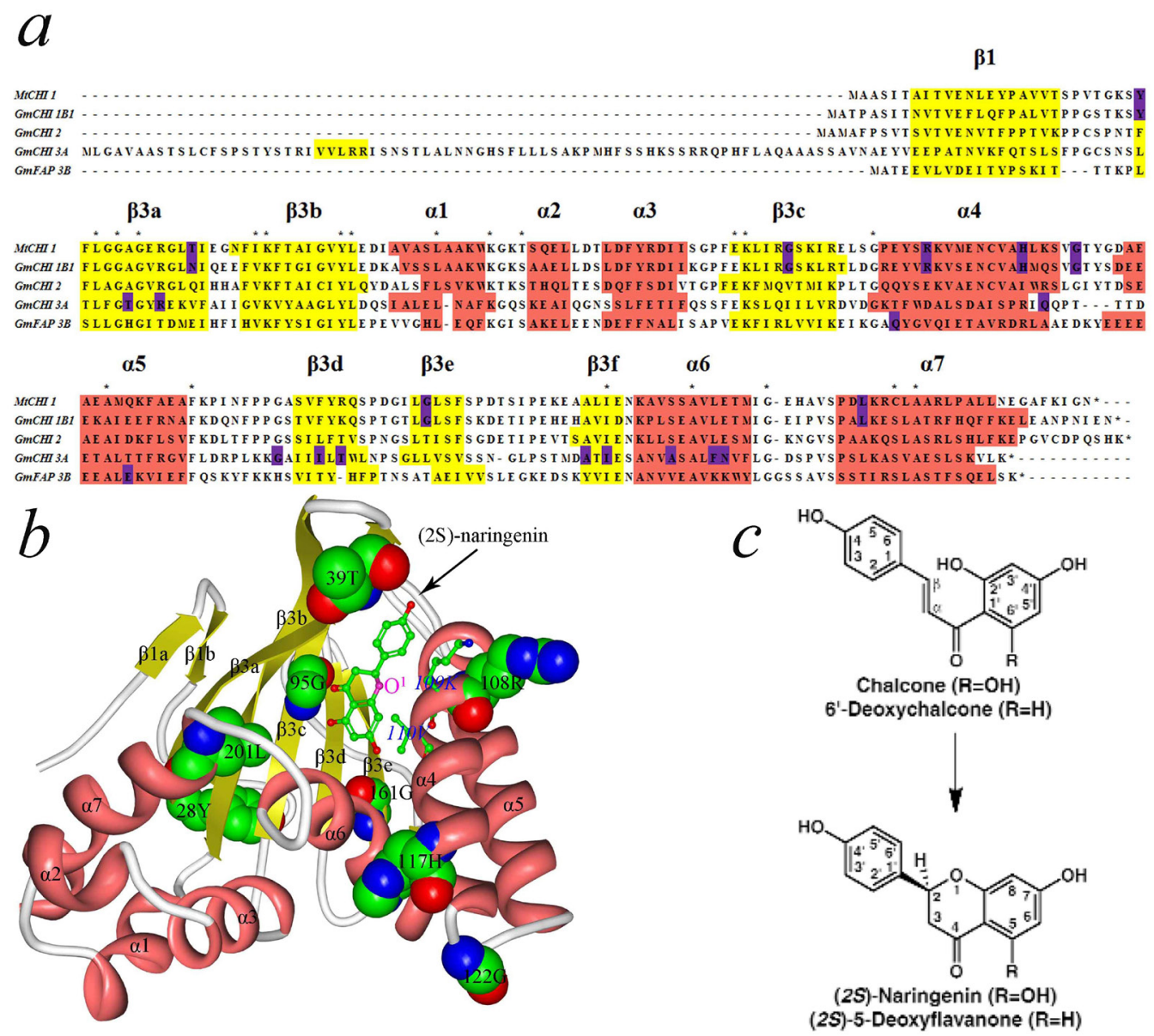

Figure 2. Structure and positively selected sites of CHI1. a. Chalcone and naringenin substrates of CHI1 are shown with their numbering systems. b. Structure of MtCHI from the Protein Data Bank that was submitted by Jez et al. (2000). The labels of $\alpha$-helices (pale red) and $\beta$-sheets (yellow) are in accordance with Jez et al. (2000). Eight positively selected sites (blue), with their molecular structures, are shown on the protein, as well as the position of (2S)-naringenin and (c) the important catalytic core constituted by $109 \mathrm{~K}$ and $110 \mathrm{~V}$. Positively selected sites are in purple, and conservative amino acid residues are marked by and asterisk $(*)$.

\section{DISCUSSION}

The $\mathrm{CHI}$ genes only exist in plants, which are involved in isoflavonoid and flavonoid synthesis; and CHIs are derived from FAP3 (Ngaki et al., 2012). The topologies of their phylogenetic trees, intron structure, and Pfam domains (Punta et al., 2012) were carefully considered to reveal the evolution of the $C H I$ gene family. There are 3 types of $C H I s ; 2$ members were identified as $\mathrm{CHI} 2$ and $\mathrm{CHI} 3$ and could be found in all of the 6 plants studied. All $\mathrm{CHI}$ and 
FAP3 proteins have similar Pfam domains, and secondary and tertiary structures. The chalcone domain identified in Pfam nearly covers all of the coding regions of $\mathrm{CHI}$, except $\mathrm{CHI}$.

Leguminous isoflavonoids are secondary metabolites that are beneficial for plant resistance to diseases and insect pests (Hegnauer and Gpayer-Barkmeijer, 1993). CHI1 is a special protein that only exists in the Leguminosae and plays an important role in the generation of Leguminosae isoflavonoids; $\mathrm{CHI1}$ is derived from CHI2 and CHI3 (Shimada et al., 2003; Ralston et al., 2005). GmCHII is highly expressed in the root and differed from the parental CHI2 and CHI3 genes, which were barely detectable in the roots (Table 3). Otherwise, the roots of the soybean can secrete isoflavonoids to stimulate soil-microbe rhizobium and form nitrogen-fixing root nodules (Verma, 1992). Thus, we suggest that CHI1 diverged from the parental CHIs; thus, CHI1 is expressed in the roots, where it has adapted to its novel role in root isoflavonoid synthesis. In correlation with the unique characteristics of CHIls in the Leguminosae, significant positive selection in the branch-site model was detected along its branch. Moreover, 8 sites with a posterior probability of $>95 \%$ were found in the BEB analysis, indicating that these amino acid of CHI1 experienced natural positive selection. In positive selection, the given sites have a larger nonsynonymous substitution rate than the synonymous substitution rate. It most often occurs under environmental changes or when plants migrate to new areas with different environmental pressures (Ridout et al., 2010). Thus, the positive selection of Leguminosae CHI1 offers a probable explanation as to why the CHI1 genes encode proteins important to Leguminosae pathogen resistance. The positively selected sites may be a primary cause of the divergence between $\mathrm{CHII}$ and the other $\mathrm{CHIs}$.

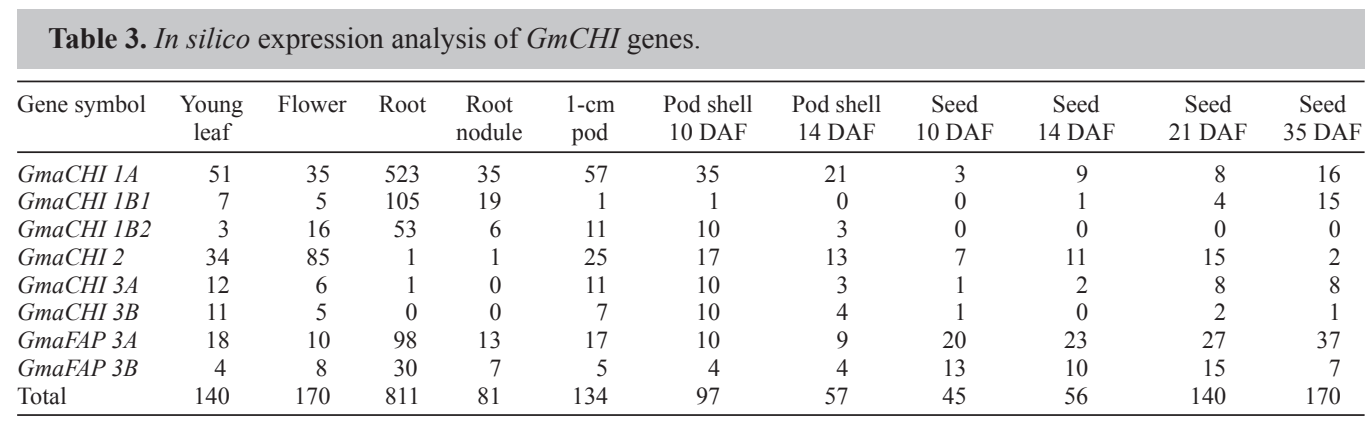

Data are the number of EST sequences matching a given DNA, with the following parameters: maximum identity $>95 \%$, length $>200$ bp and $\mathrm{E}$ value $<10^{-10}$.

The catalytic chemistry core and substrate binding play central roles during the evolution of new functions (Petsko et al., 1993; Babbitt and Gerlt, 1997; Zhang et al., 2010). The evolution of $\mathrm{CHII}$ agrees with this theory. We have found that the positively selected codons of $\mathrm{CHI} 1$ formed a bag surrounding the substrate (2S)-naringenin (Figure 2b), by the regions of $\beta 3 \mathrm{a}, \beta 3 \mathrm{c}, \alpha 4, \beta 3 \mathrm{e}$, and $\alpha 7$. CHI catalyzes on the $\mathrm{O}^{1}$ of (2S)-naringenin (Figure $2 \mathrm{c}, 2 \mathrm{~b}$ ), which is connected to $109 \mathrm{~K}$ and $110 \mathrm{~V}$ (Jez et al., 2000; Jez and Noel, 2002); interestingly the nearby site $108 \mathrm{R}$ is a positively selected site, indicating that it may modify the catalytic core of CHI1. In the tertiary structure, the $\alpha 4$ domain adjoins (2S)-naringenin, and it included 4 positively selected sites such as $108 \mathrm{R}, 117 \mathrm{H}, 109 \mathrm{~K}$, and $110 \mathrm{~V}$, as well as $122 \mathrm{G}$ (located nearby). Moreover, the side chains of some positively selected amino acids (39T, 95G, 161G, and 201L) point forward towards (2S)-naringenin. Thus, we infer that the positively selected codons, 
especially site 108R, play important roles in the function of CHI1. In addition, the evolution of CHI1 may depend on structural changes surrounding its substrate; during this process, the enzyme began to catalyze (2S)-naringenin rather than fatty acids. Indeed, (2S)-naringenin lies in the binding cleft of MtrCHII in the catalytic reaction, and the function of MtrCHII relies on the binding of the residue to the substrate (Jez et al., 2000; Jez and Noel, 2002).

\section{ACKNOWLEDGMENTS}

Research supported by the National Science Foundation of China (\#31360339), National Key Laboratory Program of Crop Genetics and Germplasm Enhancement, Nanjing Agricultural University (\#ZW2009005), Jiangxi Provincial Key Technology R\&D Program (\#20111BBF60005), Science Foundation of Jiangxi Province (\#20114BAB204022), Foundation of Jiangxi Province Educational Committee (\#GJJ12612), and Foundation of JiuJiang University (\#2012KJ09).

\section{REFERENCES}

Babbitt PC and Gerlt JA (1997). Understanding enzyme superfamilies. Chemistry as the fundamental determinant in the evolution of new catalytic activities. J. Biol. Chem. 272: 30591-30594.

Bednar RA and Hadcock JR (1988). Purification and characterization of chalcone isomerase from soybeans. J. Biol. Chem. 263: 9582-9588.

Casola C and Hahn MW (2009) Gene conversion among paralogs results in moderate false detection of positive selection using likelihood methods. J. Mol. Evol. 68: 679-687.

Ferrer JL, Austin MB, Stewart C Jr and Noel JP (2008). Structure and function of enzymes involved in the biosynthesis of phenylpropanoids. Plant Physiol. Biochem. 46: 356-370.

Guo AY, Zhu QH, Chen X and Luo JC (2007). GSDS: a gene structure display server. Yi Chuan 29: 1023-1026.

Hartmann T (2007). From waste products to ecochemicals: fifty years research of plant secondary metabolism. Phytochemistry 68: 2831-2846.

Hegnauer R and Gpayer-Barkmeijer RJ (1993). Relevance of seed polysaccharides and flavonoids for the classification of the Leguminosae: a chemotaxonomic approach. Phytochemistry 34: 3-16.

Hur S, Newby ZE and Bruice TC (2004). Transition state stabilization by general acid catalysis, water expulsion, and enzyme reorganization in Medicago savita chalcone isomerase. Proc. Natl. Acad. Sci. U S A 101: 2730-2735.

Jez JM and Noel JP (2002). Reaction mechanism of chalcone isomerase: $\mathrm{pH}$ dependence, diffusion control, and product binding differences. J. Biol. Chem. 277: 1361-1369.

Jez JM, Bowman ME, Dixon RA and Noel JP (2000). Structure and mechanism of the evolutionarily unique plant enzyme chalcone isomerase. Nature Struct. Biol. 7: 786-791.

Kosiol C, Vinar T, Fonseca RR, Hubisz MJ, et al. (2008). Patterns of positive selection in six Mammalian genomes. PLoS Genet. 4: e1000144.

Larracuente AM, Sackton TB, Greenberg AJ, Wong A, et al. (2008). Evolution of protein-coding genes in Drosophila. Trends Genet. 24: 114-123.

Ngaki MN, Louie GV, Philippe RN, Manning G, et al. (2012). Evolution of the chalcone-isomerase fold from fatty-acid binding to stereospecific catalysis. Nature 485: 530-533.

Petersen L, Bollback JP, Dimmic M, Hubisz M, et al. (2007). Genes under positive selection in Escherichia coli. Genome Res. 17: 1336-1343.

Petsko GA, Kenyon GL, Gerlt JA, Ringe D, et al. (1993). On the origin of enzymatic species. Trends Biochem. Sci. 18: 372-376.

Punta M, Coggill PC, Eberhardt RY, Mistry J, et al. (2012). The Pfam protein families database. Nucleic Acids Res. 40: D290-D301.

Ralston L, Subramanian S, Matsuno M and Yu O (2005). Partial reconstruction of flavonoid and isoflavonoid biosynthesis in yeast using soybean type I and type II chalcone isomerases. Plant Physiol. 137: 1375-1388.

Ridout KE, Dixon CJ and Filatov DA (2010). Positive selection differs between protein secondary structure elements in Drosophila. Genome Biol. Evol. 2: 166-179. 
Shimada N, Aoki T, Sato S, Nakamura Y, et al. (2003). A cluster of genes encodes the two types of chalcone isomerase involved in the biosynthesis of general flavonoids and legume-specific 5-deoxy(iso) flavonoids in Lotus japonicas. Plant Physiol. 3: 941-951.

Tamura K, Dudley J, Nei M and Kumar S (2007). MEGA4: molecular evolutionary genetics analysis (MEGA) software version 4.0. Mol. Biol. Evol. 24: 1596-1599.

Verma D (1992). Signals in root nodule organogenesis and endocytosis of rhizobium. Plant cell 4: 373-382.

Yang ZH (2007). PAML 4: phylogenetic analysis by maximum likelihood. Mol. Biol. Evol. 24: 1586-1591.

Zhang J, Yang H, Long M, Li L, et al. (2010). Evolution of enzymatic activities of testis-specific short-chain dehydrogenase/ reductase in Drosophila. J. Mol. Evol. 71: 241-249. 